Georgian Mathematical Journal

Volume 14 (2007), Number 4, 597-606

\title{
ON THE OSCILLATION OF SECOND ORDER NONLINEAR NEUTRAL DELAY DYNAMIC EQUATIONS
}

\author{
HASSAN A. AGWO
}

\begin{abstract}
In this paper we obtain some new oscillation criteria for the second order nonlinear neutral delay dynamic equation

$$
\left(x(t)-p(t) x\left(t-\tau_{1}\right)\right)^{\triangle \triangle}+q(t) f\left(x\left(t-\tau_{2}\right)\right)=0,
$$

on a time scale $\mathbb{T}$. Moreover, a new sufficient condition for the oscillation sublinear equation

$$
\left(x(t)-p(t) x\left(t-\tau_{1}\right)\right)^{\prime \prime}+q(t) f\left(x\left(t-\tau_{2}\right)\right)=0
$$

is presented, which improves other conditions and an example is given to illustrate our result.

2000 Mathematics Subject Classification: 34K11, 39A10, 39A99.

Key words and phrases: Oscillation, time scales, neutral delay, dynamic equation.
\end{abstract}

\section{INTRODUCTION}

In the recent years, the theory of time scales has received a lot of attention. This theory was introduced by S. Hilger in his Ph.D thesis in 1988 in order to unify continuous and discrete analysis (see [8]). In fact, there active research has been concerning the oscillation and nonoscillation of solutions of differential equations on time scales (or measure chains). We refer the reader to the recent papers [1], [4], [5], [12], [14], [15] and the references cited therein. A book on the subject of time scales by Bohner and Peterson [2] summarizes and organizes much of time scales calculus, see also the book by Bohner and Peterson [3] for advances in dynamic equations on time scales.

In this paper, we are concerned with the oscillation of the second order nonlinear delay dynamic equation

$$
\left(x(t)-p(t) x\left(t-\tau_{1}\right)\right)^{\triangle}+q(t) f\left(x\left(t-\tau_{2}\right)\right)=0
$$

on a time scale $\mathbb{T}$. Since we are interested in asymptotic behavior of solutions, we suppose that the time scale $\mathbb{T}$ under consideration is not bounded from the above, i.e., it is a time scale interval of the form $\left[t_{0}, \infty\right)$. on a time scale $\mathbb{T}$. $\left[t_{0}, \infty\right)_{\mathbb{T}}=\left[t_{0}, \infty\right) \cap \mathbb{T}$. Throughout the paper it is assumed that:

$\left(H_{1}\right) p(t)$ and $q(t)$ are real-valued $r d$-continuous functions defined on $\mathbb{T}$ where $0 \leq p(t)<1$ and $q(t) \geq 0$

$\left(H_{2}\right) \tau_{1}$ and $\tau_{2}$ are positive constants, $\tau_{1}<\tau_{2}$ such that the delay functions $\tau_{1}(t):=t-\tau_{1}$ and $\tau_{2}:=t-\tau_{2}$ satisfy $\tau_{1}(t): \mathbb{T} \rightarrow \mathbb{T}$ and $\tau_{2}(t): \mathbb{T} \rightarrow \mathbb{T}$ for all $t \in \mathbb{T}$. 
$\left(H_{3}\right) f: \mathbb{T} \rightarrow \mathbb{R}$ is a continuous nondecreasing function such that $u f(u)>0$ for all $u \neq 0$.

By a solution of equation (1.1) we mean a nontrivial real-value function $x(t)$ which has the properties $\left(x(t)-p(t) x\left(t-\tau_{1}\right)\right) \in C_{r d}^{2}\left[t_{x}, \infty\right), t_{x}>t_{0}$, and satisfies equation (1.1) for all $t>t_{x}$; here $C_{r d}^{2}\left[t_{x}, \infty\right)$ is the space of functions the first derivatives of which are right dense continuous on $\left[t_{x}, \infty\right)$ (see below). Our attention is restricted to those solutions of equation (1.1) which exist on some half-line $\left[t_{x}, \infty\right)$ and satisfy $\sup \left\{|x(t)|: t>t_{1}\right\}>0$ for any $t_{1}>t_{x}$.

A solution $x(t)$ of (1.1) is said to be oscillatory if it is neither eventually positive nor eventually negative. Otherwise it is called nonoscillatory. The equation itself is called oscillatory if all its solutions are oscillatory.

Note that if $\mathbb{T}=\mathbb{R}$, we have $\sigma(t)=\rho(t)=t, \mu(t)=0, f^{\Delta}(t)=f^{\prime}(t)$ and in that case equation (1.1) becomes the second-order nonlinear neutral delay differential equation

$$
\left(x(t)-p(t) x\left(t-\tau_{1}\right)\right)^{\prime \prime}+q(t) f\left(x\left(t-\tau_{2}\right)\right)=0, \quad t \in\left[t_{0}, \infty\right),
$$

which has been investigated by many authors, see, for example, [6], [7], [9]-[11].

Wong in [13] proves that when $p(t) \equiv p$, the necessary and sufficient for the sublinear equation (1.2) to oscillate is

$$
\int^{\infty} q(t) f(t) d t=\infty
$$

However, Lin in [10] claims that the sufficient condition of Wong in the above result may turn out to be false, but Lin does not give a counterexample to show that his claim is true and proves that equation (1.2) is oscillatory if

$$
\int^{\infty} q(t) d t=\infty
$$

holds in a sublinear case.

In this paper, we obtain a new sufficient condition for equation (1.1) to oscillate and a new sufficient condition for the sublinear equation (1.2) to oscillate, which improves Lin condition. Moreover, we give an example to illustrate our result.

\section{Some Preliminaries on Time Scales}

A time scale $\mathbb{T}$ is an arbitrary nonempty closed subset of the real numbers $R$. On any time scale $T$, we define the forward and the backward jump operators by

$$
\sigma(t):=\inf \{s \in \mathbb{T}: s>t\} \quad \text { and } \quad \rho(t):=\sup \{s \in \mathbb{T}: s<t\},
$$

respectively.

A point $t \in \mathbb{T}, t>\inf \mathbb{T}$ is said to be left-dense if $\rho(t)=t$, right-dense if $t<\sup \mathbb{T}$ and $\sigma(t)=t$, left-scattered if $\rho(t)<t$ and right-scattered if $\sigma(t)>t$. 
The graininess function $\mu: \mathbb{T} \rightarrow[0, \infty)$ is defined by $\mu(t):=\sigma(t)-t$. For the function $f: \mathbb{T} \rightarrow \mathbb{R}$ the (delta) derivative is defined by

$$
f^{\triangle}(t):=\frac{f(\sigma(t))-f(t)}{\sigma(t)-t} ;
$$

$f$ is said to be differentiable if its derivative exists. A useful formula is

$$
f^{\sigma}:=f(\sigma(t))=f(t)+\mu(t) f^{\triangle}(t),
$$

If $f, g$ are differentiable, then $f g$ and the quotient $\frac{f}{g}$ (where $g g^{\sigma} \neq 0$ ) are differentiable with

$$
(f g)^{\triangle}=f^{\triangle} g+f^{\sigma} g^{\triangle}=f g^{\triangle}+f^{\triangle} g^{\sigma}
$$

and

$$
\left(\frac{f}{g}\right)^{\triangle}:=\frac{f^{\triangle} g-f g^{\triangle}}{g g^{\triangle}} .
$$

If $f^{\triangle}(t) \geq 0$, then $f$ is nondecreasing.

A function $f:[a, b] \rightarrow \mathbb{R}$ is said to be right-dense continuous if it right continuous at each right-dense point and there exists a finite left limit at all left-dense points. A function $f: \mathbb{T} \rightarrow \mathbb{R}$ is called regressive if $1+\mu(t) f(t) \neq 0$ for all $t \in \mathbb{T}$. The set of all functions $f: \mathbb{T} \rightarrow \mathbb{R}$ which are regressive and $r d$-continuous will be denoted by $C_{r}$. We define the set $\mathbb{R}^{+}$of all positively regressive elements of $\mathbb{R}$ by $\mathbb{R}^{+}=\{f \in \mathbb{R}: 1+\mu(t) f(t) \neq 0, t \in \mathbb{T}\}$. A function $F$ with $F^{\triangle}=f$ is called an antiderivative of $f$ and then we define

$$
\int_{a}^{b} f(t) \triangle t=F(b)-F(a)
$$

where $a, b \in \mathbb{T}$. It is well known that $r d$-continuous functions possess antiderivatives. A simple consequence of formula (2.3) is

$$
\int_{t}^{\sigma(t)} f(s) \triangle s=\mu(t) f(t),
$$

and improper integrals defined in the usual way

$$
\int_{a}^{\infty} f(t) \triangle t=\lim _{b \rightarrow \infty} \int_{a}^{b} f(t) \triangle t .
$$

If $p \in \mathbb{R}$, then the exponential function is defined as

$$
e_{p}(t, s)=\exp \left(\int_{s}^{t} \xi_{\mu(\nu)}(p(\nu) \triangle \nu),\right.
$$


for all $s, t \in \mathbb{T}$, where $\xi_{h}(z)$ is the cylinder transformation given by

$$
\xi_{h}(z):= \begin{cases}\frac{\log (1+h z)}{h}, & h \neq 0, \\ z, & h=0 .\end{cases}
$$

For the properties of the exponential function we refer to [2].

\section{Main Results}

In this section, we investigate the oscillation of equation (1.1).

Theorem 3.1. Assume that $\left(H_{1}\right)-\left(H_{3}\right)$ holds and there exists $\ell \in[0,1)$ such that $0 \leq p(t) \leq \ell<1$ for large $t$. Then every solution of equation (1.1) oscillates, if the inequality

$$
z^{\triangle}(t)+\frac{\varepsilon}{\ell} q(t) f(z(t-\delta)) \leq 0, \quad \varepsilon>0,
$$

where $\delta=\tau_{2}-\tau_{1}-\varepsilon$ for any $0<\varepsilon<\tau_{2}-\tau_{1}$, has no eventually positive solution.

Proof. Suppose to the contrary that equation (1.1) has a nonoscillatory solution $x(t)$. We may assume without loss of generality that $x(t-\rho)>0$ where $\rho=$ $\max \left\{\tau_{1}, \tau_{2}\right\}$ and $0 \leq p(t) \leq \ell<1$ for all $t>t_{0}$. Set

$$
y(t)=x(t)-p(t) x\left(t-\tau_{1}\right)
$$

Then from equation (1.1) it follows that

$$
y^{\triangle}(t)=-q(t) f\left(x\left(t-\tau_{2}\right)\right) \leq 0 \text { for all } t>t_{0} .
$$

This shows that $y^{\Delta}(t)$ is nonincreasing on $\left[t_{0}+\rho, \infty\right)$. Hence there are two possible cases:

(a) $y^{\Delta}(t)>0$ for all $t \geq t_{0}+\rho$ or

(b) $y^{\Delta}(t)<0$ for all $t \geq t_{1}$.

If the second case holds, i.e., $y^{\Delta}(t)<0$ for all $t \geq t_{1}$, then we have

$$
y(t)-y\left(t_{0}\right)=\int_{t_{0}}^{t} y^{\Delta}(s) \triangle s \leq y^{\Delta}\left(t_{0}\right)\left(t-t_{0}\right)
$$

and consequently $y(t) \rightarrow-\infty$ as $t \rightarrow \infty$, which implies that there exist $c>0$ and $t_{2}>t_{1}$ such that $y(t)<-c$ for all $t>t_{2}$. Then $x(t) \leq-c+\ell x\left(t-\tau_{1}\right)$ for all $t>t_{2}$, which implies that

$$
x\left(t_{2}+n \tau_{1}\right) \leq-c \sum_{i=0}^{n-1} \ell^{i}+\ell^{n} x\left(t_{2}\right)
$$

and so, $x\left(t_{2}+n \tau_{1}\right)<0$ for large $n$, which contradicts the fact that $x(t)>0$ for all $t \geq t_{0}$. Hence $y^{\Delta}(t)>0$ for all $t \geq t_{0}+\rho$. This shows that $y(t)$ is increasing on $\left[t_{0}+\rho, \infty\right)$ and so there are two possible cases:

(i) $y(t)<0$ for all $t \geq t_{0}+\rho$ or

(ii) $y(t)>0$ for all $t \geq t_{3}$ for some $t_{3} \geq t_{0}$. 
If case (i) holds, i.e., $y(t)<0$ for all $t \geq t_{0}+\rho$, then

$$
y(t)>-p(t) x\left(t-\tau_{1}\right)>-\ell x\left(t-\tau_{1}\right)
$$

which implies that

and then

$$
x\left(t-\tau_{1}\right)>-\frac{1}{\ell} y(t)
$$

$$
x\left(t-\tau_{2}\right)>-\frac{1}{\ell} y\left(t+\tau_{1}-\tau_{2}\right), \quad t \geq t_{2}+2 \rho .
$$

Since $f$ is nondecreasing,

$$
f\left(x\left(t-\tau_{2}\right)\right)>f\left(-\frac{1}{\ell} y\left(t+\tau_{1}-\tau_{2}\right)\right), \quad t \geq t_{2}+2 \rho,
$$

which implies that

$$
y^{\triangle}(t)+q(t) f\left(-\frac{1}{\ell} y\left(t+\tau_{1}-\tau_{2}\right)\right), \quad t \geq t_{2}+2 \rho .
$$

Integrating inequality (3.6) from $t \geq t_{0}+\rho$ to $\infty$, we obtain

$$
-y^{\triangle}(t)+\int_{t}^{\infty} q(s) f\left(-\frac{1}{\ell} y\left(s+\tau_{1}-\tau_{2}\right)\right) \triangle s \leq 0 .
$$

Since $y(t)$ is increasing on $\left[t_{0}+\rho, \infty\right),-y(t)$ is decreasing and therefore for any $0<\varepsilon<\tau_{2}-\tau_{1}$ we have

$$
-y^{\triangle}(t)+f\left(-\frac{1}{\ell} y\left(t+\tau_{1}-\tau_{2}\right)\right) \int_{t}^{t+\varepsilon} q(s) \triangle s \leq 0 .
$$

Set

$$
z(y)=-\frac{1}{\ell} y(t), \quad \delta=\tau_{2}-\tau_{1}-\varepsilon \quad \text { and } \quad Q(t)=\frac{1}{\ell} \int_{t}^{t+\varepsilon} q(s) \triangle s .
$$

Then

$$
z^{\triangle}(t)+Q(t) f(z(t-\delta)) \leq 0 .
$$

This shows that inequality (3.9) has an eventually positive solution $z(t)$, which contradicts the assumption of our theorem. This contradiction shows that case (i) is impossible.

If case (ii) holds, i.e., $y(t)>0$ and $y^{\triangle}(t)>0$ for all $t \geq t_{3}$ and for some $t_{3} \geq t_{0}$, then $x(t) \geq y(t)$ and consequently from equation (1.1) it follows that

$$
y^{\triangle}(t)+q(t) f\left(y\left(t-\tau_{2}\right)\right) \leq 0, \quad t \geq t_{3}+\rho .
$$

Then

$$
\begin{aligned}
y(t) & =y\left(t_{4}\right)+\int_{t_{4}}^{t} y^{\triangle}(s) \triangle s>\int_{t_{4}}^{t} y^{\triangle}(s) \triangle s \\
& >\left(t-t_{4}\right) y^{\triangle}(t)>\frac{\varepsilon}{\ell} y^{\triangle}(t) \text { for all } t>\frac{\varepsilon}{\ell}+t_{4} .
\end{aligned}
$$


Since

$$
y\left(t-\tau_{2}\right)>\frac{\varepsilon}{\ell} y^{\triangle}\left(t-\tau_{2}\right) \geq \frac{\varepsilon}{\ell} y^{\triangle}\left(t-\tau_{2}+\tau_{1}+\varepsilon\right),
$$

we have

$$
f\left(y\left(t-\tau_{2}\right)\right) \geq f\left(\frac{\varepsilon}{\ell} y^{\triangle}\left(t-\tau_{2}+\tau_{1}+\varepsilon\right)\right) .
$$

Set

$$
w(t)=\frac{\varepsilon}{\ell} y^{\triangle}(t) .
$$

Now from (3.10), (3.12) and (3.13) we get

$$
w^{\triangle}(t)+Q(t) f(w(t-\delta)) \leq 0,
$$

where $\delta=\tau_{2}-\tau_{1}-\varepsilon, 0<\varepsilon<\tau_{2}-\tau_{1}$, which has an eventually positive $w(t)$. This is a contradiction and so case (ii) is impossible. The proof is complete.

Theorem 3.2. Assume that

$$
z^{\triangle}(t)+Q(t) f(z(t-\delta)) \leq 0,
$$

where $Q(t)>0, f$ is a nondecreasing function such that $u f(u)>0$ for all $u \neq 0$, and that

$$
0<\int_{0}^{t_{0}} \frac{\triangle s}{f(s)}, \quad \int_{-t_{0}}^{0} \frac{\triangle s}{-f(s)}<\infty \quad \forall t_{0}>0 .
$$

Then all solutions of (3.14) are oscillatory if

$$
\int^{\infty} Q(s) \triangle s=\infty
$$

Proof. Suppose to the contrary that inequality (3.14) has a nonoscillatory solution $z(t)$. We may assume without loss of generality that $z(t-\delta)>0$ for all $t \geq t_{0}+\delta$. Then from (3.14) it follows that

$$
z^{\triangle}(t) \leq-Q(t) f(z(t-\delta))
$$

This shows that $z(t)$ is nonincreasing on $\left[t_{0}+\rho, \infty\right)$. Hence we can write

$$
Q(t) \leq-\frac{z^{\triangle}(t)}{f(z(t))} \quad \text { for all } t \geq t_{0}+\delta
$$

Integrating inequality (3.18) from $t_{1}=t_{0}+\delta$ to $t$, we obtain

$$
\begin{aligned}
\int_{t_{1}}^{t} Q(s) \triangle s & \leq-\int_{t_{1}}^{t} \frac{z^{\triangle}(t)}{f(z(t))} \triangle s=-\int_{z\left(t_{1}\right)}^{z(t)} \frac{\triangle s}{f(s)}=\int_{0}^{z\left(t_{1}\right)} \frac{\triangle s}{f(s)}-\int_{0}^{z(t)} \frac{\triangle s}{f(s)} \\
& <\int_{0}^{z\left(t_{1}\right)} \frac{\triangle s}{f(s)}<\infty
\end{aligned}
$$

which is a contradiction, since the left side of of the above inequality tends to $\infty$ as $t \rightarrow \infty$. 
Theorem 3.1 reduces the question of oscillation of equation (1.1) to the absence of an eventually positive solution (oscillatory) of the differential inequality (3.14). As a consequence Theorem 3.1 and Theorem 3.2 immediately imply

Theorem 3.3. Assume that $\left(H_{1}\right)-\left(H_{3}\right)$ holds and there exists $\ell \in[0,1)$ such that for large $t, 0 \leq p(t) \leq \ell<1$ and $0<\int_{0}^{t_{0}} \frac{\triangle s}{f(s)}, \int_{-t_{0}}^{0} \frac{\triangle s}{-f(s)}<\infty \forall t_{0}>0$. Then every solution of equation (1.1) oscillates if

$$
\int^{\infty} q(s) \triangle s=\infty .
$$

Theorem 3.4. Assume that $f$ is a nondecreasing function such that $|f(u v)|>$ $f(u) f(v), u f(u)>0$ for all $u \neq 0$, and that

$$
0<\int_{0}^{t_{0}} \frac{\triangle s}{f(s)}, \int_{-t_{0}}^{0} \frac{\triangle s}{-f(s)}<\infty \quad \forall t_{0}>0 .
$$

If there exists $\alpha(t)>0, \alpha^{\triangle}(t)>0$, such that

$$
\int^{\infty} \frac{Q(s)}{\alpha(s)} f(\alpha(s-\delta)) \triangle s=\infty,
$$

then all solutions of (3.14) are oscillatory.

Proof. As in the proof of Theorem (3.1) we get $z(t)>0, z(t-\delta)>0$ and $z^{\triangle}<0$ for all $t>t_{0}+\delta$.

Let

$$
z(t)=u(t) \alpha(t)
$$

where $u, \alpha>0, u^{\triangle}<0$ and $\alpha^{\triangle}>0$ for all $t>t_{0}+\delta$. Then

$$
z^{\triangle}(t)=u^{\triangle} \alpha+u^{\sigma} \alpha^{\triangle}
$$

and, consequently,

$$
u^{\triangle}(t) \alpha(t)+u(\sigma(t)) \alpha^{\triangle}(t)+Q(t) f(\alpha(t-\delta)) f(u(t-\delta)) \leq 0,
$$

which implies that

$$
u^{\triangle}(t)+\frac{Q(t)}{\alpha(t)} f(\alpha(t-\delta)) f(u(t-\delta)) \leq 0 .
$$

Following the procedure as in Theorem 3.2 the statement of this theorem follows.

Theorem 3.5. Assume that $f$ is a nondecreasing function such that $f(u v)>$ $f(u) f(v)$ if $u v \geq 0, u f(u)>0$ for all $u \neq 0$ and that

$$
0<\int_{0}^{t_{0}} \frac{\triangle s}{f(s)}, \int_{-t_{0}}^{0} \frac{\triangle s}{-f(s)}<\infty \quad \forall t_{0}>0 .
$$


If there exists $\alpha(t)>0, \alpha^{\triangle}(t)>0$, such that

$$
\int^{\infty} \frac{q(s)}{\alpha(s)} f(\alpha(s-\delta)) \triangle s=\infty
$$

then all solutions of (1.1) are oscillatory.

Theorem 3.6. Assume that $f$ is a nondecreasing function such that $|f(u v)|>$ $f(u) f(v), u f(u)>0$ for all $u \neq 0$. Then all solution of (1.1) are oscillatory if

$$
\int^{\infty} q(s) f\left(\left(1+p\left(s-\tau_{2}\right)\right) f\left(\frac{s-\beta}{2}\right) \triangle s=\infty, \quad \beta=\tau_{1}+\tau_{2} .\right.
$$

Proof. Suppose to the contrary that $x(t)$ is a nonoscillatory solution of equation (1.1) and proceed as in Theorem 3.1 to get $y^{\triangle}(t)>0$ for $t \geq t_{0}+\rho$ where $\rho=\max \left\{\tau_{1}, \tau_{2}\right\}$. Then $y(t)$ is an increasing function on $\left[t_{0}+\delta, \infty\right)$.

Since $y(t)=x(t)-p(t) x\left(t-\tau_{1}\right)$, we have

$$
\begin{aligned}
x(t) & =y(t)+p(t) x\left(t-\tau_{1}\right) \\
& =y(t)+p(t)\left(y\left(t-\tau_{1}\right)+p\left(t-\tau_{1}\right) x\left(t-2 \tau_{1}\right)\right) \\
& >y(t)+p(t) y\left(t-\tau_{1}\right) \geq(1+p(t)) y\left(t-\tau_{1}\right)
\end{aligned}
$$

and

$$
\begin{aligned}
y(t) & =y\left(t_{1}\right)+\int_{t_{1}}^{t} y^{\triangle}(s) \triangle s>\int_{t_{1}}^{t} y^{\triangle}(s) \triangle s \\
& >\left(t-t_{1}\right) y^{\triangle}(t)>\frac{t}{2} y^{\triangle}(t) \text { for all } t>\rho+t_{1} .
\end{aligned}
$$

Then

$$
x(t)>(1+p(t))\left(\frac{t-\tau_{1}}{2}\right) y^{\triangle}\left(t-\tau_{1}\right),
$$

which implies that

$$
y^{\triangle}(t)+q(t) f\left(\left(1+p\left(t-\tau_{2}\right)\right)\left(\frac{t-\left(\tau_{1}+\tau_{2}\right)}{2}\right)\right) f\left(y^{\triangle}\left(t-\left(\tau_{1}+\tau_{2}\right)\right) \leq 0 .\right.
$$

Putting

$$
z(t)=y^{\triangle}(t),
$$

inequality (3.26) can be rewritten as

$$
z^{\triangleright}(t)+Q(t) f(z(t-\beta)) \leq 0,
$$

where

$$
Q(t)=q(t) f\left(1+p\left(t-\tau_{2}\right)\right) f\left(\frac{t-\beta}{2}\right), \quad \beta=\tau_{1}+\tau_{2},
$$

has a positive solution, which is a contradiction. 
Remark 3.1. We note when $\mathbb{T}=\mathbb{R}$, equation (1.1) becomes the second order nonlinear neutral differential equation (1.2) and condition (3.19) becomes

$$
\int^{\infty} q(s) d s=\infty
$$

which coincides with Lin's condition (1.4), while condition (3.24) takes the form

$$
\int^{\infty} \frac{q(s)}{\alpha(s)} f(\alpha(s-\delta) d s=\infty .
$$

Also, condition (3.25) takes the form

$$
\int^{\infty} q(s) f\left(\left(1+p\left(s-\tau_{2}\right)\right) f\left(\frac{s-\beta}{2}\right) d s=\infty, \beta=\tau_{1}+\tau_{2} .\right.
$$

Remark 3.2. Lin in [10] claims that the sufficient condition (1.3) for the oscillation of sublinear equation (1.2) may be false and proves that condition (1.4) is sufficient for the oscillation of such equations. It is clear that condition(3.25) improves Lin's condition and corrects the sufficient condition of Wong.

In the following, an example is given to illustrate our conclusion.

Example. Consider the following second-order sublinear neutral differential equation

$$
\left.\left(x(t)-p x\left(t-\tau_{1}\right)\right)^{\prime \prime}+q(t)\left|x\left(t-\tau_{2}\right)\right|^{\nu} \operatorname{sgn} x\left(t-\tau_{2}\right)\right)=0, \quad 0<\nu<1,
$$

where $0<p<1$ and $q(t)=t^{-3 \nu}$. Then

$$
\int_{T}^{\infty} q(t) d t= \begin{cases}\infty, & 0<\nu \leq \frac{1}{3} \\ \text { finite, } & \frac{1}{3}<\nu<1\end{cases}
$$

This shows that equation(3.31) is oscillatory when $0<\nu \leq \frac{1}{3}$ according to Lin condition (1.4) and condition (3.28). But

$$
\int_{T}^{\infty} q(t) f(t) d t= \begin{cases}\infty, & 0<\nu \leq \frac{1}{2} \\ \text { finite, }, & \frac{1}{2}<\nu<1\end{cases}
$$

This shows that equation (3.31) is oscillatory when $0<\nu \leq \frac{1}{3}$ according to Wong's condition (1.4).

Thus we have

$$
\int_{T}^{\infty} q(s) f\left(1+p\left(s-\tau_{2}\right)\right) f\left(\frac{s-\beta}{2}\right) d s= \begin{cases}\infty, & 0<\nu \leq \frac{1}{2+\alpha}, \quad \alpha>0, \\ \text { finite, } & \frac{1}{2}<\nu<1 .\end{cases}
$$

Therefore that condition(3.25) improves Lin's condition and corrects the sufficient condition of Wong. 


\section{REFERENCES}

1. R. P. Agarwal, D. O'Regan, and S. H. Saker, Oscillation criteria for second-order nonlinear neutral delay dynamic equations. J. Math. Anal. Appl. 300(2004), No. 1, 203217.

2. M. Bohner and A. Peterson, Dynamic equations on time scales. An introduction with applications. Birkhäuser Boston, Inc., Boston, MA, 2001.

3. M. Bohner and A. Peterson, Advances in dynamic equations on time scales. Birkhäuser Boston, Inc., Boston, MA, 2003.

4. L. Erbe, A. Peterson, and S. H. SAKer, Oscillation criteria for second-order nonlinear dynamic equations on time scales. J. London Math. Soc. (2) 67(2003), No. 3, 701-714.

5. L. Erbe and A. Peterson, Boundedness and oscillation for nonlinear dynamic equations on a time scale. Proc. Amer. Math. Soc. 132(2004), No. 3, 735-744 (electronic).

6. L. Erbe, Q. Kong, and B. G. Zhang, Oscillation theory for functional-differential equations. Monographs and Textbooks in Pure and Applied Mathematics, 190. Marcel Dekker, Inc., New York, 1995.

7. I. GYÖRI and G. LADAS, Oscillation theory of delay differential equations. With applications. Oxford Mathematical Monographs. Oxford Science Publications. The Clarendon Press, Oxford University Press, New York, 1991.

8. S. Hilger, Analysis on measure chains - a unified approach to continuous and discrete calculus. Results Math. 18(1990), No. 1-2, 18-56.

9. J. JIANG and X. LI, Oscillation of second order nonlinear neutral differential equations. Appl. Math. Comput. 135 (2003), No. 2-3, 531-540.

10. X. Lin, Oscillation of second-order nonlinear neutral differential equations. J. Math. Anal. Appl. 309(2005), No. 2, 442-452.

11. Y. ŞAHINER, On oscillation of second order neutral type delay differential equations. Appl. Math. Comput. 150(2004), No. 3, 697-706.

12. S. H. SAKer, Oscillation of nonlinear dynamic equations on time scales. Appl. Math. Comput. 148(2004), No. 1, 81-91.

13. J. S. W. Wong, Necessary and sufficient conditions for oscillation of second order neutral differential equations. J. Math. Anal. Appl. 252(2000), No. 1, 342-352.

14. B. G. Zhang and X. Deng, Oscillation of delay differential equations on time scales. Math. Comput. Modelling 36(2002), No. 11-13, 1307-1318.

15. B. G. Zhang and ZH. Shanliang, Oscillation of second-order nonlinear delay dynamic equations on time scales. Comput. Math. Appl. 49(2005), No. 4, 599-609.

(Received 5.09.2005)

Author's address:

Department of Mathematics

Ain Shams University, Faculty of Education

Roxy, Cairo

Egypt

E-mail: Hassanagwa@yahoo.com 